Ann. Biol. anim. Bioch. Biophys., I974, 14 (3), 47 I-486.

\title{
INDIVIDUALITÉ MUSCULAIRE CHEZ LE BOVIN : ÉTUDE DE L'ÉQUIPEMENT ENZYMATIQUE DE QUELQUES MUSCLES
}

\author{
M. ANSAY \\ avec la collaboration technique de E. Laurent et de J. Roupain \\ Chaive de Génétique, Faculté de Médecine vétérinaire, \\ 45, Rue des Vétérinaires, \\ 1070 Bruxelles (Belgique)
}

\section{RÉSUMÉ}

Nous avons étudié divers critères biochimiques permettant de différencier 9 muscles du veau de 83 kilos. Classés dans l'ordre croissant de leur activité Phosphoglycéraldéhyde déhydrogénase (GAPDH), ce sont : le cœur, le masséter, le diaphragme, le Splenius, le Flexor digitorum superficialis, le Psoas major, le Semitendinosus, le Longissimus dorsi, le Biceps temoris.

L'aldolase totale (ALD), la phosphorylase (Pyase), la créatine phosphokinase (CPK), la lactate déhydrogénase (LDH), la fraction de l'aldolase liée (ALD B/B + C) sont d'autres caractéristiques biochimiques permettant de classer ces divers muscles dans le même ordre.

D'autres critères classent les muscles dans un ordre exactement inverse : les activités G6PD + 6PGD, GOT, la concentration en DNA-P, les rapports $\mathrm{MDH} / \mathrm{LDH}, \mathrm{MDH} / \mathrm{GAPDH}$, le rapport isozymique pour la LDH (basse concentration en pyruvate/haute concentration en pyruvate).

La signification de ces caractéristiques biochimiques du point de vue de la fonction de ces muscles et du point de vue du problème de l'hypertrophie musculaire des bovidés est également discutée.

\section{INTRODUC'TION}

Que le tissu musculaire dans sa fonction principale, la transformation de l'énergie chimique en énergie mécanique, soit l'objet de spécialisations est une observation déjà très ancienne. Enn I874, RANVIFR écrivait : "L'action des deux espèces de muscles n'étant pas la même, il est possible que leur rôle est différent; les pâles, avec leur contraction brusque, seraient des muscles d'action par excellence; les rouges, avec leur contraction plus lente et plus persistante, seraient plutôt équilibrateurs ou régulateurs. " 
C'était là, en physiologie musculaire, pour la première fois, tenter de relier un caractère de coloration (" rouge ou pâle ") à la qualité d'une fonction (contraction brusque ou lente).

D'un point de vue physiologique, la distinction de RANviER entre muscles lents et muscles rapides demeure entièrement valable. Cependant la couleur (muscle blanc, muscle rouge), résultat de la teneur en divers pigments héminiques, n'est pas en exacte corrélation avec le type physiologique (BARNARD et al., I97I ; BURLEIGH et SCHIMKE. r969).

I,a vitesse de contraction d'un muscle donné est caractéristique : au niveau biochimique, elle est directement liée à l'activité ATPasique de la myosine (BARANY et al., I965; BARANY, I967 ; MOMMAERTS, I970). Elle est également sous contrôle nerveux ; l'innervation croisée de deux muscles à temps de contraction très différent produit de profonds changements tant dans les caractéristiques physiologiques de ces muscles (BULLER et al., I960) que dans leurs propriétés biochimiques (MommaERTS et al., I969 ; PREWI'T' et SALAFSKy, I970 ; Golisch et al., I970).

En histologie musculaire, les coupes transversales de fibres soumises à divers réactifs présentent des réactions typiques, variables d'une fibre à l'autre, de façon discontinue. La variété des réactifs employés et des réponses observées a conduit les auteurs à proposer un grand nombre de systèmes de classification, non entièrement superposables (fibres blanches, rouges ou intermédiaires, fibres de type I ou II, fibres de type A, B... H, fibres $\alpha \mathrm{R}, \alpha \mathrm{W}, \beta \mathrm{R}$ ) (Dubowitz, I970 ; DAwson et RomanuL, r964; Ashmore et DoERr, I97I ; STEIN et PADYkULA, I962).

Le type d'une fibre comporte des implications importantes notamment au niveau du devenir de cette fibre : le développement postnatal d'un muscle s'accomplit principalement par le grandissement préférentiel des fibres dites "blanches " (COOPER et al., I970 ; SWATLAND et CASSENS, I972) ou par la transformation de fibres "rouges" en fibres " blanches " (Ashmore et al., I972), éventuellement par dilution du contenu mitochondrial de la fibre (Goldspink, I969).

D'un point de vue économique, les questions de CASSENs (I970) : "La croissance musculaire, la quantité de muscle fourni sont des choses importantes. Résultent-elles d'une hypertrophie de type II ? Cette hypertrophie peut-elle être contrôlée?", trouvent des prolongements dans l'inégalité de susceptibilité des fibres blanches et rouges à l'hypertrophie musculaire chez les bovidés (AsHMORE et RoBINSON, I969), à la dystrophie musculaire chez le poulet (Cosmos, I970), à la viande exsudative chez le porc (BRIsKEY, I964).

Les muscles lents (" rouges )) et les muscles rapides (“ blancs ») diffèrent également par nombre de caractéristiques biochimiques. Au niveau des protéines myofibrillaires, on retrouve des différences qualitatives entre les deux muscles (SARKaR et al., I97I ; PARsons et al., I969). Les muscles " rouges " ont une activité de synthèse protéique plus importante (DREYFUs, I967 ; SHORT, I969 ; GoldBrERG, I967). Cependant, la caractéristique la mieux connue de ces deux muscles repose sur le mode de leur approvisionnement en énergie : la fibre lente (" rouge ") dépend surtout du métabolisme oxydatif et est ainsi capable d'une activité prolongée et soutenue. Le muscle " blanc ", avec une capacité élevée de glycolyse, est bien adapté à des mouvements brusques et volontaires (BURLEIGH et SchImke, I968, I969 ; BASs et al., I969).

Au cours de l'ontogenèse, la fonction mitochondriale évolue assez peu dans les 
muscles, qu'ils soient " blancs" ou " rouges ". Par opposition, la fonction glycolytique s'élève très rapidement dans les muscles "blancs ", mais demeure à un niveau plus stable dans les muscles " rouges " (BASs et al. r970; CoOper et al., I97 I ANSAY, I972; MANN et SALAFsky, I970; DALRYMPLE et al., I973).

Peu de travaux ont exploité, à la fois, dans le domaine histologique et biochimique, les mêmes méthodes. BEATTY et al. (1966) ont cependant montré une corrélation étroite entre les données résultant d'une classification qualitative histochimique et les mesures quantitatives d'activité basées sur un même enzyme : la succino-déhydrogénase.

Au cours de ce travail, nous comparons neuf muscles du bovin en utilisant divers critères de différenciation biochimique (BAss et al., I969; BEATTY et BocEK, I970). Ces muscles se classent dans un ordre assez caractéristique, ascendant ou descendant, suivant le paramètre employé.

Parmi les enzymes utilisés, certains : la phosphoglycéraldéhyde déhydrogénase (GAPDH), l'hexokinase (HK), la phosphorylase (PYASE), la lactate déhydrogénase $(\mathrm{L} D \mathrm{DH})$ sont les enzymes " clés " (key enzymes) et représentent ici, respectivement, la glycolyse, la phosphorylation du glucose, la glycogénolyse, la fermentation lactique (BAss et al. I969).

L'aldolase possède la propriété intéressante de s'absorber de manière réversible à l'actine (ARNold et PETTE, I968; ANSAY, I974).

La créatine phosphokinase (CPK) assure l'interconvertibilité de deux formes différentes d'énergie chimique musculaire : l'ATP et la créatine phosphate.

Deux enzymes, la malate déhydrogénase (MDH) et la glutamique oxaloacétique transaminase (GOT) ont une distribution cytoplasmique et mitochondriale reposant sur l'existence de deux isozymes aux propriétés différentes (ANSAY, I973). La part respective revenant à chacun de ces deux isoenzymes peut être estimée par extraction différentielle en tampon sucrose et en tampon phosphate (PETTE, I968).

La concentration en DNA-P est une bonne mesure de la concentration en noyaux (Durand et al., I968). Pendant le développement du muscle fœtal, la concentration en DNA-P et l'activité combinée de la glucose-6-phosphate déhydrogénase et de la 6-phosphogluconate déhydrogénase (G6PD + 6PGD) évoluent de manière parallèle (ANSAY, I974).

La proportion dans la lactate déhydrogénase (LDH) de sous-unités " $\mathrm{H}$ " est en étroite corrélation avec le rapport isozymique ou rapport des activités LDH estimées à basse concentration en pyruvate et à haute concentration en pyruvate (WILSON et al. 1963).

\section{MATÉRIEL, ET TECHNIQUES EXPÉRIMENTALES}

\section{A. - Les animaux}

Les déterminations portent sur six veaux de race de Moyenne et Haute Belgique : deux culards d'un âge moyen de 28 jours (23 et 33 ) et quatre veaux normaux d'un âge moyen de $5^{8}$ jours $(45$. 54,67 et 67 ); 3 mâles et 3 femelles. Ils ont été abattus au poids constant de 83 kilos (HAxSET et ANSAY, 1972) et disséqués suivant la technique de BuTTERFIELD et MAY (I966). 


\section{B. - Homogénéisation}

L'échantillon prélevé (endéans les deux heures suivant l'abattage) dans « le gros et au milieu du muscle " et conservé dans l'azote liquide est additionné à raison de $9 \mathrm{ml}$ par g, d'un tampon appelé "tampon saccharose " et ainsi constitué : saccharose $0,3 \mathrm{M}$; Triéthanolamine Io $\mathrm{mM}$; EDTA $2 \mathrm{mM}$; 2-mercaptoéthanol I5 mM; pH 7,2 (PETTE, I968). Il est ensuite homogénéisé à froid, à l'aide d'un appareil "Ultra-Turrax ", à vitesse ménagée pendant I 5 secondes. Cette opération fournit un homogénat total dont une partie est destinée au dosage du DNA (en triple exemplaire : $3 \times 2,5 \mathrm{ml}$ ) (MUNRo et FLECK, I966; ANSAY et GILlET, 1973) ou après incubation (I heure a $0^{\circ} \mathrm{C}$ ) avec un volume égal de Triton 2 p. Ioo à la mesure de l'activité hexokinasique (Burleigh et Schimke, i968). Le surnageant de centrifugation de l'autre partie (35000 $g$; 20 minutes; $0-5^{\circ} \mathrm{C}$ ) sert à la mesure d'une première série d'activités enzymatiques (série " $\mathrm{B}$ ") comprenant les enzymes ou isozymes extramitochondriaux et la fraction "libre ": CPK, PYASE, GAPDH, ALD, GOT, G6PD + 6PGD, LDH Haute, $_{\text {LDH }}$ Basse).

Le culot de centrifugation est repris à une dilution identique, par du "tampon phosphate " $\left(\mathrm{Na}_{2} \mathrm{HPO}_{4} / \mathrm{KH}_{2} \mathrm{PO}_{4}\right.$ o, I M, 2-mercaptoéthanol I5 $\left.\mathrm{mM}, \mathrm{pH} 7,25\right)$ et réhomogénéisé deux fois 30 secondes à vitesse maximum (ANSAY, 1974). A partir du surnageant on mesure une seconde série d'activités enzymatiques (série " $\mathrm{C}$ » comprenant les enzymes ou isozymes intramitochondriaux et la fraction "liée ": GAPDH, ALD, MDH, GOT, LDH.)

Ce schéma constitué par deux homogénéisations successives dans deux tampons différents est inspiré de la méthode de PetTe (I968).

\section{C. - Mesure des activités enzymatiques}

Les principes généraux ont été décrits dans un mémoire précédent (ANSAY, 1974).

I. Hexokinase (E.C. 2.7.I.I. HK).

Triéthanolamine $50 \mathrm{mM}$ pH 7.6; EDTA $5 \mathrm{mM}$; Glucose $25 \mathrm{mM}$; $\mathrm{MgCl}_{2}$ Io $\mathrm{mM}$; NADP o,3 mM ; Glucose-6-phosphate déhydrogénase $0,7 \mathrm{U} / \mathrm{ml}$; Adénosine triphosphate $3,6 \mathrm{mM}$; NaF $33 \mathrm{mM}$.

2. Créatine phosphokinase (E.C. 2.7.3.2. CPK).

Avec le test TC-V, no 5992 TCAE de Boehringer.

3. Phosphorylase (E.C. 2.4.I.1. Pyase) (Bass et al., I969).

Triéthanolamine $50 \mathrm{mM}$ pH 7,6 ; EDTA $5 \mathrm{mM} ; \mathrm{MgCl}_{2}$ Io $\mathrm{mM} ; \mathrm{KH}_{2} \mathrm{PO}_{4} / \mathrm{Na}_{2} \mathrm{HPO}_{4}$ Io $\mathrm{mM} \mathrm{pH} 7,6$; L-cystéine $\mathrm{HCl}$ neutralisée extemporanément Io $\mathrm{mM}$; NADP o, $3 \mathrm{mM}$; AMP 2 mM ; Glycogène o,5 p. Ioo ; Phosphoglucomutase I U/ml ; Glucose 6-phosphate déhydrogénase $0,7 \mathrm{U} / \mathrm{ml}$; Glucose-I-6-phosphate $0,05 \mathrm{mM}$.

4. Phosphoglycéraldéhyde déhydrogénase (E.C. I.2.I.I2 GAPDH).

Triéthanolamine $50 \mathrm{mM} \mathrm{pH} 7,6$; EDTA $5 \mathrm{mM} ; \mathrm{MgCl}_{2} 0,33 \mathrm{mM} ; \mathrm{NADH}_{2} 0,234 \mathrm{mM}$; 3-phosphoglycérate $3,5 \mathrm{mM}$; Phosphoglycératekinase $3 \mathrm{U} / \mathrm{ml}$; Adénosine triphosphate $3,6 \mathrm{mM}$.

5. Aldolase (E.C. 4.I.2.I3 ALD).

Triéthanolamine $50 \mathrm{mM} \mathrm{pH} \mathrm{7,6} \mathrm{;} \mathrm{EDTA} 5 \mathrm{mM}$; $\mathrm{NADH}_{2}$ o, $234 \mathrm{mM}$; mélange Glycérophosphatedéhydrogénase/Phosphotrioseisomérase $27 \mu \mathrm{g} / \mathrm{ml}$; Fructose I-6-diphosphate $3,5 \mathrm{mM}$.

6. Lactate déhydrogénase (E.C. I.I.I.27 LDH) (Fox et REED, I969).

Triéthanolamine $50 \mathrm{mM}$ pH 7,6; EDTA $5 \mathrm{mM} ; \mathrm{NADH}_{2} 0,234 \mathrm{mM}$;

Haute concentration en pyruvate : pyruvate ro $\mathrm{mM}$.

Basse concentration en pyruvate : pyruvate $0,33 \mathrm{mM}$.

7. Glucose 6-phosphate déhydrogénase (E.C. I.I.I.49 G6PD) + 6-phosphogluconate déhydrogénase (E.C. I.I.I.44 6PGD) (Glock et McLeAn, I953) Triéthanolamine 50 mM pH 7,6 ; EDTA $5 \mathrm{mM}$; NADP o,3 mM ; Glucose-6-phosphate 3.5 mM ; 6-phosphogluconate $3,5 \mathrm{mM}$. 
8. Malate déhydrogénase (E.C. I.I.I.37 $\mathrm{MDH}$ ).

Tampon phosphate $\mathrm{pH} 7,4$ o,I M; $\mathrm{NADH}_{2}$ o,234 mM; Acide aspartique $36 \mathrm{mM}$; $\alpha$-cétoglutarate $1,67 \mathrm{mM}$; Glutamique oxaloacétique transaminase $3 \mathrm{U} / \mathrm{ml}$.

9. Glutamique oxaloacétique transaminase (E.C. 2.6.I.I. GOT).

Tampon phosphate $\mathrm{pH} 7,4 \mathrm{O}, \mathrm{I} \mathrm{M}$; acide aspartique $125 \mathrm{mM} ; \mathrm{NADH}_{2} \quad 0,234 \mathrm{mM}$; $\alpha$-cétoglutarate $6,7 \mathrm{mM}$; mélange $\mathrm{MDH} / \mathrm{LDH}$ du test Boehringer TCA II 15.955 TGAF $\mathrm{r} 2,5 \mu \mathrm{g} / \mathrm{ml}$; blanc sans acide aspartique.

\section{D. — Enzymoélectrophorèse}

L'enzymoélectrophorèse de quelques extraits musculaires (fig. 3) a été conduite selon une méthode générale précédemment décrite (ANSAY, I973) en utilisant comme support d'électrophorèse une bande de Cellogel.

Les zones d'activité sont révélées suivant SHaw et Prasad (r970).

\section{RÉSUL'TA'TS}

I. L'analyse de la variance à deux voies (" entre muscles " et " entre individus ») a montré que toutes les différences entre muscles étaient très hautement significatives. En ce qui concerne les différences entre animaux, les enzymes suivants montraient une différence significative au seuil de 5 p. Ioo : 1'aldolase, la CPK, la phosphorylase, la GOT.

2. L'extraction en milieu saccharose extrait $90 \mathrm{p}$. Ioo de la $\mathrm{L}$ DH, $54 \mathrm{p}$. Ioo de la GOT et $76 \mathrm{p}$. Ioo de la $\mathrm{MDH}$ mesurables. Ces valeurs ne varient pas significativement d'un animal à l'autre ou d'un muscle à l'autre (tabl. I).

\section{TABLEAU I}

Pourcentage de l'activité enzymatique totale extraite en tampon saccharose (6 animaux, moyenne \pm écart-type)

\begin{tabular}{|c|c|c|c|c|c|}
\hline & GAPDH & ALD & $\mathrm{LDH}_{\text {Basse }}$ & GOT & $\mathrm{MDH}$ \\
\hline Coeur & $39,7+10,9$ & $8,0 \pm \quad 7,3$ & $94,6 \pm 1,6$ & $48,7 \pm 7,1$ & $77,5 \pm 4,6$ \\
\hline Masseter & $51,6 \pm 14,6$ & $15,3 \pm \quad 5,8$ & $90,2 \pm \quad 3,7$ & $62,7 \pm 3,5$ & $71,8 \pm 9,0$ \\
\hline Diaphragme ......... & $42,5 \pm 10,9$ & $31,7 \pm 24,4$ & $88,9 \pm \quad 4,9$ & $48,5 \pm \quad 7,0$ & $73,6 \pm 5,7$ \\
\hline Splenius ............ & $50,0 \pm 12,3$ & $28,5 \pm 7,9$ & $85,8 \pm 9,1$ & $46,9 \pm \quad 7,8$ & $73,7 \pm 9,4$ \\
\hline $\begin{array}{l}\text { Flexor digitorum super- } \\
\quad \text { ficialis } \ldots \ldots \ldots \ldots \ldots \ldots\end{array}$ & $50,9 \pm 13,5$ & $38,2 \pm 8,4$ & $89,5 \pm 2,5$ & $57,0 \pm 6,4$ & $75,5 \pm 7,6$ \\
\hline Psoas major ........... & $54,8 \pm 10,2$ & $26,4 \pm 8,2$ & $89,4 \pm 1,4$ & $57,7 \pm 12,6$ & $82,8 \pm 3,1$ \\
\hline Semi-tendinosus .... & $51,8 \pm 10,6$ & $53,9 \pm 13,9$ & $89,8 \pm 1,3$ & $53,5 \pm \quad 9,1$ & $80,7 \pm 5,0$ \\
\hline Longissimus dorsi & $60,8 \pm 7,1$ & $58,5 \pm 8,9$ & $85,8 \pm 12,0$ & $52,0 \pm 13,0$ & $70,2 \pm 6,3$ \\
\hline Biceps femoris .... & $68,7 \pm 9,9$ & $66,5 \pm 14,4$ & $91,6 \pm 1,9$ & $56,4 \pm 8,7$ & $79,9 \pm 4,5$ \\
\hline
\end{tabular}

Le chiffre de go p. Ioo, pour la $\mathrm{LDH}$, mesure le rendement d'extraction d'un enzyme normalement presque entièrement situé dans le cytosol (94 à $9^{8} \mathrm{p}$. Ioo) (PeTTE, I968; DE DOMENECH et al. I970). 
Le pourcentage d'aldolase extraite est très différent d'un muscle à l'autre et certaines de ces différences sont hautement significatives (tabl. $2,2^{\text {e }}$ partie). La GAPDH paraît se conformer à une même distribution et montre une extractibilité faible pour le cour et le diaphragme ( $\pm 40 \mathrm{p}$. Ioo), plus élevée (60 à $70 \mathrm{p}$. Ioo) pour les $m$. biceps femoris et longissimus dorsi. Cependant, le masseter, muscle typiquement " rouge ", ne libère en tampon saccharose pas plus d'enzyme que le muscle semitendinosus.
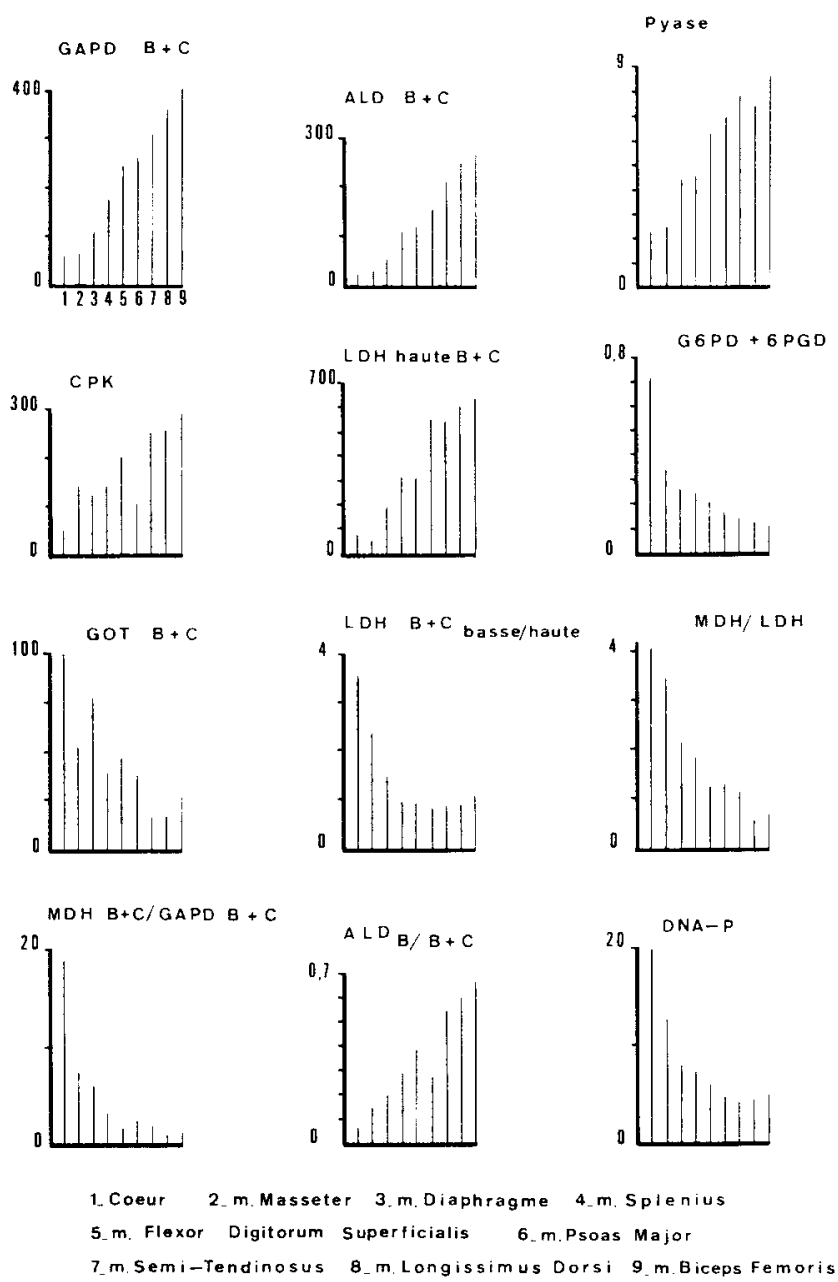

FIG. I. - Critères biochimiques permettant une bonne caractérisation de divers muscles du bovin Dans tous les schémas, les muscles sont classés suivant l'ordre de leur activité GAPDH

3. Dans la figure $I$, on a classé tous les muscles sur la base de leur activité GAPDH totale. Le muscle biceps femoris (40I U par g de poids frais) possède une activité 6 à 7 fois plus élevée que le cœur (6r U par g de poids frais).

Pour l'aldolase, la phosphorylase, la créatine phosphokinase, la lactate déhydrogénase, le rapport de l' " aldolase liée " à l'aldolase totale ( $\mathrm{ALD} B / \mathrm{B}+\mathrm{C})$, ces mêmes 


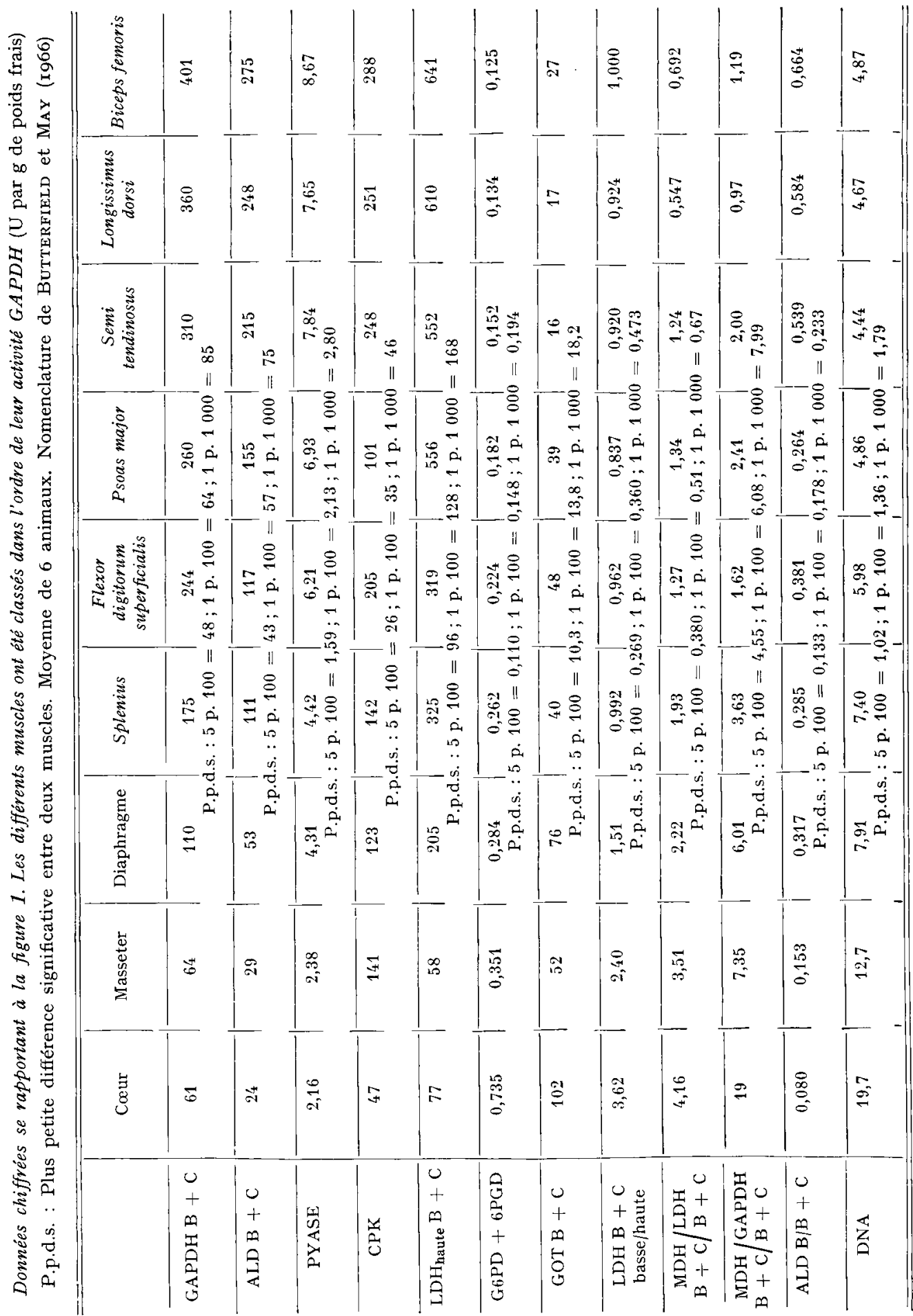

Annales de Biologie animale. - 1974 . 
muscles montrent un comportement d'ensemble identique. Les activités les plus basses sont celles du cœur ; elles sont légèrement plus élevées pour le masseter et le diaphragme mais culminent à leur valeur maximum pour les 3 plus gros muscles : m. semi-tendinosus, m. longissimus dorsi, m. biceps femoris.

Pour une série d'autres critères, le comportement est tout à fait opposé avec des valeurs maximales pour le cœur, minimales pour les gros muscles. Il s'agit des activités G6PD + 6PGD, GOT totale, de la concentration en DNA-P, du rapport isozymique pour la $\mathrm{LDH}$ (rapport de l'activité à " basse " sur l'activité à " haute " concentration en pyruvate) des rapports $\mathrm{MDH} / \mathrm{LDH}, \mathrm{MDH} / \mathrm{GAPDH}$.

Dans le tableau 2, les mêmes données sont rapportées sous forme chiffrée. En même temps, on a calculé la plus petite différence significative aux seuils de $5 \mathrm{p}$. Ioo, I p. IOo, I p. IOoo qui permette de distinguer deux muscles l'un de l'autre.
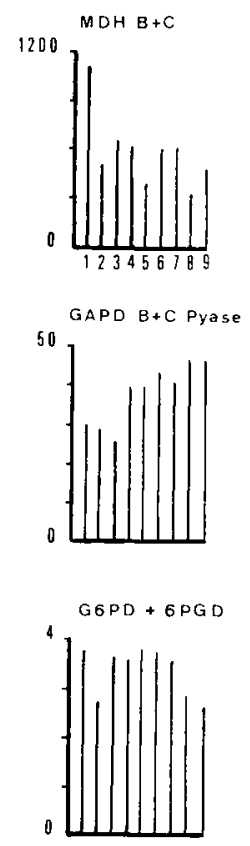

Fig. 2. - Critères biochimiques ne permettant pas une bonne individualisation de divers muscles du bovin L'ordre des muscles est identique à celui de la figure I

Dans la figure 2 sont présentés certains critères biochimiques peu utiles à la différenciation musculaire telle qu'elle est présentée dans le tableau $\mathbf{I}$ ou dans la figure I.

a) L’activité MDH élevée dans le coeur ne différencie cependant pas le masseter et le $\mathrm{m}$. biceps femoris.

b) L'azote total extrait $(B+C)$ est plus important dans les gros muscles que dans le cœur mais il n'y a pas de différence entre le $m$. psoas par exemple et le cour.

c) Certains rapports entre activités enzymatiques encore appelés " rapports non discriminants " (BASS et al. I 969) ne montrent guère de différence d'un muscle à l'autre (CPK/Pyase) (GAPDH/Pyase). 
d) Enfin, la référence choisie pour exprimer une activité enzymatique a également son importance puisque 1'activité G6PD + 6PGD exprimée par mg de DNA-P (ANSAY, I974) ne montre pas de différences d'un muscle à l'autre.

5. L'hexokinase, un enzyme lié pour une large part aux structures cellulaires (ANDERSON et al., I97I), notamment aux mitochondries, est plus élevée dans le cour et le masseter que dans les gros muscles (tabl. 3).

A ce comportement d'ensemble, il y a cependant deux exceptions notables : le diaphragme avec un taux relativement bas et le muscle psoas caractérisé par une activité enzymatique à peine mesurable.

\section{TABLEAU 3}

Activité de l'hexokinase musculaire extraite en présence de Triton à 1 p. 100 (en $U$ par $g$ de poids frais; 6 animaux : moyenne \pm écart-type)

\begin{tabular}{|c|c|c|c|}
\hline$\ldots \ldots \ldots \ldots$ & $2,37+0,58$ & Psoas major & $0,12 \neq 0,07$ \\
\hline Masseter........... & $1,88 \pm 0,53$ & Longissimus dorsi & $0,89 \pm 0,55$ \\
\hline Diaphragme ........ & $0,96 \pm 0,30$ & Semi-tendinosus .... & $1,19 \mp 0,70$ \\
\hline Splenius ........... & $1,22 \pm 0,37$ & Biceps femoris ...... & $1,01 \pm 0,49$ \\
\hline $\begin{array}{l}\text { Flexor digitorum super- } \\
\text { ficialis } \ldots \ldots \ldots \ldots\end{array}$ & $1,55 \pm 0,25$ & & \\
\hline
\end{tabular}

\section{DISCUSSION}

\section{Signification des critères biochimiques utilisés}

Le concept de l'individualité musculaire fondé sur un équipement enzymatique différent remonte probablement, chez les espèces domestiques, aux travaux de LAWRIE (I953). Chez le cheval adulte, classés dans l'ordre d'activité enzymatique croissante (cytochrome oxydase, succinate déhydrogénase), on trouvait le m. longissimus dorsi suivi du m. psoas, du diaphragme et du cour.

Actuellement, cette différenciation enzymatique peut être détectée à de multiples niveaux. PetTre et son école (PETTE, I966, I968; BASs et al., I969, I970; Golish et al. I970; HOFER et al. I97I) ont bien montré l'existence de groupes d'enzymes à l'intérieur desquels les enzymes individuels gardent, quelle que soit leur valeur absolue, une même relation l'un par rapport à l'autre (groupes enzymatiques à proportion constante). L'estimation de l'activité d'un enzyme peut être ainsi indicatrice de l'activité d'un segment typique du métabolisme. Rapportés l'un à l'autre, ces enzymes " clés " définissent des " relations de systèmes " constantes ou variables : en d'autres termes, leur activité relative est indépendante de leur activité absolue.

D'une espèce animale à l'autre, d'un muscle à l'autre, le rapport phosphorylase GAPDH s'avère être très constant. Par contre, le rapport phosphorylase/hexokinase varie dans les mêmes conditions de I à 50 (BURLEIGH et SchIMKE, Ig68; Bass et al., rg69).

Un exemple de la signification physiologique de ces relations de systèmes est donné par le rapport constant de la glycérol-I-phosphate déhydrogénase mitochon- 
driale à la glycéraldehyde phosphate dehydrognéase (Kraus, I967 ; BAss et al., r969). L'activité de l'enzyme glycérol-I-phosphate déhydrogenase (composante du glycérolphosphate shuttle) est précisément un des nombreux moyens d'oxyder le $\mathrm{NADH}_{2}$ produit par la glycolyse (Wu et al. I969; LEHNINGER, I97I). Les muscles blancs à activité glycolytique plus élevée ont également une activité $\alpha$-glycérophosphate déhydrogénase plus marquée (BLANCHAER, I964; BASs et al. I969).

I. Les enzymes représentatifs de la glycolyse (GAPDH et ALD), de la fermentation lactique (la $\mathrm{LDH}$ ), de la glycogénolyse (la phosphorylase), la CPK ont un comportement d'ensemble très symétrique (fig. I). Ils sont étroitement correlés et sont, l'un comparé à l'autre, dans un rapport constant.

SIGEI, et PET'TE (I969) ont montré que les enzymes de la glycolyse et de la glycogénolyse étaient localisés dans le muscle dans des zones très bien circonscrites, avec des concentrations locales très élevées, au niveau des bandes isotropiques (actine). Ces enzymes ne seraient pas à l'état de solution mais de gel et organisés en fonction de la séquence des réactions catalysées. L'idée, remarquent AMBERson et BAUER (I97I) que les enzymes glycolytiques sont librement dissous dans le sarcoplasme est maintenant abandonnée.

2. Le problème de l'absorption de l'aldolase, et dans une moindre mesure, de la GAPDH, aux protéines myofibrillaires a souvent été abordé (ARNOLD et PETTE, I968 ; STARI.INGER, I967 ; AmbERSOn et BAUER, I97I). L'augmentation de la force ionique (ARNOLD et PETTE, I968; ANSAy, I974), 1'action de divers métabolites (Fructose r.6. diphosphate) (ARNOLD et PETTE, I970) sont des facteurs capables de relâcher les liens unissant l'aldolase et l'actine. Cette liaison qui peut modifier les propriétés catalytiques de l'enzyme (ARNOLD et PETTE, I970 ; ChARKE et al., I970) est encore accrue dans les muscles qui ont été préalablement stimulés (STARLINGER, I967). Cette dernière observation cadre bien avec la constatation rapportée plus haut, que le pourcentage d'aldolase liée est le plus grand dans les muscles " rouges ", muscles à activité soutenue.

3. Le rapport des activités $\mathrm{LDH}$ à basse et à haute concentration en pyruvate (OPIE et NEWSHOLME, I967; MARGRETH et al., I970) est une indication des proportions relatives de sous-unités isozymiques $H$ et $M$. Un rapport élevé traduit une

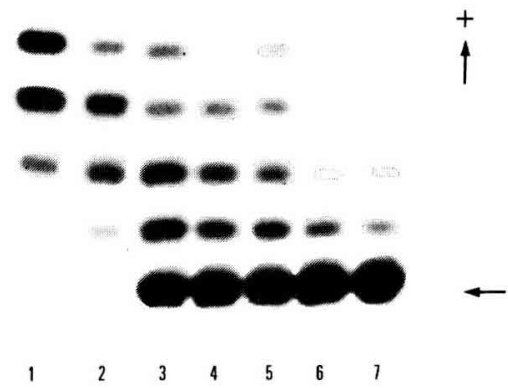

FIG. 3. - Zymogramme de la LDH de divers muscles appartenant à des veaux de 83 kilos Électrophorèse sur Cellogel (240 V, I h 30), (ANSAY, I973)

Dans l'ordre décroissant de migration anodique, les isozymes $\mathrm{H}_{4}, \mathrm{H}_{3} \mathrm{M}_{2} \mathrm{H}_{2} \mathrm{M}_{2}, \mathrm{HM}_{3}, \mathrm{M}_{4}$.

De gauche à droite : le cour, le masséter, le diaphragme, l'Obliquus capitis caudalis, le Splenius, le Flexor digitorum longus, le Biceps femoris. 
contribution prépondérante de 1'isozyme $\mathrm{H}$ (WILSON $e t$ al., I963 ; Fox et REED, I969). La valeur de 3,62 pour le ventricule du cœur de veau que nous rapportons est intermédiaire entre les valeurs de 4,15 pour le cour de rat (TURner et Manchester, I972), 2,90 pour l'homme, 2,70 pour le chien (Fox et REED, I969).

La figure 3 se rapportant à un zymogramme LDH effectué à partir de quelques muscles d'un de ces veaux confirme cette interprétation d'une proportion de moins en moins grande des sous-unités $H$ en allant du cœur vers les gros muscles.

4. Le tampon saccharose extrait quelque $76 \mathrm{p}$. roo de la $\mathrm{MDH}$ totale (tabl. I) : cette valeur, assez constante d'un muscle à l'autre et reflétant l'importance de la malate déhydrogénase cytoplasmique, peut varier en fonction de l'état physiologique de l'organe : elle est de 58,6 p. Ioo dans l'utérus normal, de 74,9 p. Ioo dans l'utérus gravide (GEYER, I968).

La $\mathrm{MDH}$ du $\mathrm{m}$. longissimus dorsi du porc est à l'état complètement soluble ; dans les autres muscles, $5^{\circ} \mathrm{p}$. Ioo de la $\mathrm{MDH}$ totale est localisée dans le sarcoplasme (EI-BADAwI et HaMm, I972). Pour les mêmes auteurs, il n'y a pas de corrélation entre la $\mathrm{MDH}$ et le contenu en myoglobine de divers muscles de la vache : le $\mathbf{m}$. longissimus dorsi montre une activité supérieure à celle du diaphragme. MANN et Salafsky (I970) ont montré, chez le chat, que l'activité des enzymes oxydatifs, peu différents chez les muscles rapides ou lents, était déjà fixée à la naissance.

5. Pour 1'hexokinase, nos résultats (Tableau 3) corroborent les conclusions de Burleigh et SChImke (I968, r969), de CRABTREe et Newshorme (I972) à savoir que l'utilisation du glycogène est moins importante et l'utilisation du glucose plus importante dans les muscles " rouges" que dans les muscles "blancs". PETER et al. (I968) avaient également remarqué que l'hexokinase était plus active dans les muscles " rouges " que dans les muscles " blancs ". Il y a chez 1'homme normal une étroite corrélation entre les niveaux d'activité de l'hexokinase et de la citrate synthase (HoFkR et al., I97I). Des résultats préliminaires de ce laboratoire montrent une pareille corrélation chez le bovin.

6. Le contenu en DNA-P diminue en allant du cour vers le muscle biceps femoris. Le contenu en G6PD + 6PGD subit une évolution parallèle : cette association étroite avait déjà été notée au cours de l'ontogenèse du $\mathrm{m}$. biceps femoris du fœetus bovin (ANSAY, I974).

7. Les muscles rouges, dont le pouvoir de synthèse protéique est plus important, ont une concentration en RNA plus élevée (GoLDBERG, I967).

Au confluent du métabolisme des hydrates de carbone et des protéines, la GOT totale apparaît plus élevée dans les muscles rouges que dans les muscles blancs (PetTe, I960; Opie et Newsholme, I967). Chez la vache, les observations de Hamm (I969) sont ainsi confirmées : chez les deux animaux examinés, le diaphragme et le muscle extensor carpi radialis démontraient des activités GOT plus élevées que les muscles longissimus dorsi, pectoralis superficialis et $p$ soas.

Le pourcentage moyen de la GOT extraite en tampon saccharose et correspondant à 1'activité de la GOT cytoplasmique est de 53,7 p. Ioo, assez peu variable d'un muscle à l'autre. Pour cinq muscles du porc, HAMM (I969) rapporte un chiffre correspondant égal à $46,8 \mathrm{p}$. Ioo. 


\section{Propriétés de certains muscles et équipement enzymatique}

Pour tous les critères employés, le cœur présente les valeurs les plus basses (glycolyse, glycogénolyse, fermentation lactique, CPK) ou les plus élevées (voie des pentoses, concentration en DNA, degré de liaison de l'aldolase, rapport isozymique pour la $\mathrm{LDH}, \mathrm{GO} T$, rapports $\mathrm{MDH} / \mathrm{LDH}, \mathrm{MDH} / \mathrm{GAPDH}$ ).

Le masseter présente beaucoup de similitude avec le cœur. Peu étudié dans les autres espèces animales, c'est un muscle caractérisé chez les ruminants, par des mouvements lents et continus.

De par les critères biochimiques employés, le diaphragme paraît le plus souvent nettement moins " rouge " que le cœur ou le masseter. Son activité hexokinasique, particulièrement, est équivalente à celle d'un gros muscle comme le biceps femoris. Pour BuRleirgh et Schimke (I969), le diaphragme de bœuf présente une activité hexokinasique très faible, à peine plus élevée que celle du muscle semitendinosus du lapin. Les comparaisons interspécifiques apportent d'utiles indications. GAUTHIER (I970) a montré que la composition et la taille de la fibre musculaire du diaphragme étaient proportionnelles à la grandeur de l'animal. Chez un petit mammifère, les fibres sont petites et riches en mitochondries. Le diaphragme d'un gros animal serait, au contraire, composé presque entièrement de très grandes fibres blanches. BURL.EIGH et Scmimke (I969) ont relevé que le taux d'activité hexokinasique du cour ou du diaphragme était relié à la fréquence du rythme cardiaque ou respiratoire. Pour ce muscle, il n'y a pas de relation étroite entre la teneur en myoglobine et l'activité hexokinasique.

Le m. splenius, un muscle du cou, occupe dans l'échelle adoptée une position intermédiaire. Récemment (ANSAY, I974) nous avons montré que plusieurs muscles du cou se distinguaient par une concentration en DNA-P très élevée.

Dans la race étudiée, ces muscles ont la propriété de réagir aux substances androgènes par une forte hypertrophie. Cette hypertrophie de la région cervicale se retrouve chez le taureau adulte et à un degré souvent extrême, chez le très jeune fœus mâle.

Chez le porc, le muscle trapezius est catalogué comme muscle " rouge " et démontre à ce titre, un équipement enzymatique (GOT élevée, phosphorylase basse) et une composition isozymique pour la LDH très caractéristiques (BEECHER et al., I965; Moody et CASSENS, I968; CoOper et al., I97I).

Seul représentant des muscles des extrémités digitées, le $\mathrm{m}$. flexor digitorum superficialis occupe également un classement intermédiaire. Étudié par rapport aux muscles du tronc chez l'homme, l'ensemble de ces muscles démontre une activité glycolytique moindre et une activité respiratoire en général plus élevée (LAUDAHN et al., I968).

La principale caractéristique du muscle psoas est une activité hexokinasique anormalement faible, Io fois moins élevée que celle du muscle semi-tendineux (tabl. 3). SIGEL et PETTE (I969) mentionnent également que le muscle psoas du lapin a une très faible concentration en hexokinase.

Les trois autres muscles (semitendinosus, longissimus dorsi et biceps femoris) sont de gros muscles situés le long du rachis ou dans les segments supérieurs des membres. Tous les critères employés démontrent que le métabolisme oxydatif y est faible et qu'ils présentent un grand nombre de caractéristiques de la fibre "blanche ". 
Il est intéressant de remarquer que chez le culard, ces trois muscles comptent parmi les plus hypertrophiés (Rolinss et al. I969; BocCARD et DUMONT, I972; Dumont et Boccard, I972; HANSET et ANSAy, I972). Réciproquement, ce sont les muscles dont l'hypertrophie relative est la plus faible (muscles du cou et des extrémités digitées) chez le culard, qui présentent la concentration en DNA-P la plus élevée (ANSAY, 1974) et ont une glycolyse comparativement plus faible.

Reçu pour publication en décembre 1973.

\title{
REMERCIEMEN'TS
}

Ce travail a été exécuté sous les auspices de l'Institut pour l'encouragement de la Recherche Scientifique dans l'Industrie et l'Agriculture (I. R. S. I. A.).

\section{SUMMARY}

\author{
MUSCULAR INDIVIDUALITY IN CATTLE : \\ STUDY OF THE ENZYMATIC EQUIPMENT OF SOME MUSCLES
}

We studied various biochemical criteria by which we could define 9 muscles of the 83 -kilo calf. Classified in increasing order of phosphoglyceraldehyde dehydrogenase (GAPDH) activity, they are : heart, masseter, diaphram, Splenius, Flexor digitorum superficialis, Psoas major, Semitendinosus, Longissimus dorsi, Biceps femoris.

Total aldolase (ALD), phosphorylase (Pyase), creatin phosphokinase (CPK), Iactate dehydrogenase (LDH), and the fraction of the bound aldolase (ALD B/B $+C$ ) are other biochemical characteristics which may be used to classify various muscles in the same order.

Other criteria classify muscles in an exactly inverse order : G6PD + 6PGD activities, GOT, DNA-P concentration, the ratios $\mathrm{MDH} / \mathrm{LDH}, \mathrm{MDH} / \mathrm{GAPDH}$, the isozymic ratio for LDH (low pyruvate concentration/high pyruvate concentration).

The significance of these biochemical characteristics are also discussed in regard to the functioning of these muscles and the problem of muscular hypertrophy in cattle.

\section{RÉFÉRENCES BIBLIOGRAPHIQUES}

Amberson W. R., Bauer A., i97r. Electrophoretic studies of muscle proteins. II. Complex formation between deltaprotein, myogen and protein. J. Cell. Physiol., 77, 281-300.

Anderson J. W., Herman R. H., Tyrrell J. B., Cohn R. M., ig7i. Hexokinase : a compartmented enzyme. Amer. J. Clin. Nutr., 24, 642-650.

Ansay M., I972. Essai de caractérisation biochimique du muscle de bovins culards. Ann. Génét. Sél. anim., 4, rзo-13I.

ANSAY M., 1973. Variabilité génétique et tissulaive de la malate déhydrogénase mitochondriale (MOR), de la transaminase glutamique oxaloacétique cytoplasmique (GOT), de la phosphoglucomutase (PGM), de l'adénosine déaminase (ADA), de la purine nucléoside phosphorylase (NP) dans l'espèce bovine. Thèse d'agrégation de l'enseignement supérieur (Université de Liège).

ANSAY M., 1974. Individualité musculaire chez le bovin : étude de la concentration en DNA de quelques muscles. Ann. Biol. anim. Bioch. Biophys., 14, 575-578. 
Ansay M., 1974. Développement biochimique du muscle de fœtus bovin. III. Enzymes et différenciation. Ann. Biol. anim. Bioch. Biophys., 14, I05-I 6 .

Ansay M., Gillet A., I973. Étude biochimique comparée du tissu musculaire des bovidés culards et normaux, après la naissance. I. Les acides nucléiques et l'azote total. Ann. Méd. Vét., 117, 391-400.

Arnold H., Pette D., r968. Binding of glycolytic enzymes to structure proteins of the muscle. Eur. J. Biochem., 6, 163-171.

Arnold H., Pette D., I97o. Binding of aldolase and triosephosphate dehydrogenase to F. Actin and modification of catalytic properties of aldolase. Eur. J. Bioch., 15, 360-366.

Ashmore C. R., Robinson D. W., r969. Hereditary muscular hypertrophy in the bovine. I. Histological and biochemical characterization. Proc. Soc. Exptl. Biol. Med., 132, 548-554.

Ashmore C. R., Doerr L., I97I. Postnatal development of fiber types in normal and dystrophic skeletal muscle of the chick. Exp. Neurol., 30, 43 I-446.

Ashmore C. R., Thompkin G., Doerr L., I972. Postnatal development of muscle fiber types in domestic animals. J. Anim. Sci., 34, 37-4I.

Barany M., Barany K., Reckard T., Volpe A., I965. Myosin of fast and slow muscles of the rabbit. Arch. Bioch., Biophys., 109, 185-19I.

Barany M., I967. ATPase activity of myosin correlated with speed of muscle shortening. "The contractil process" Symposium. J. Gener. Physiol., 50, suppl., 197-2 18.

Barnard R. J., Edgerton V. R., Furukawa T., Peter J. B., I971. Histochemical, biochemical and contractile properties of red, white and intermediate fibers. Amer. J. Physiol., 220, 4I0-4I4.

Bass A., Brdiczka D., Eyer P., Hofer S., Pette D., Ig69. Metabolic differentiation of distinct muscle types at the level of enzymatic organization. Eur. J. Biochern., 10, 198-206.

Bass A., Lusch G., Pette D., r97o. Postnatal differentiation of the enzyme activity pattern of energy-supplying metabolism in slow (red) and fast (white) muscles of chicken. Eur. J. Bioch., 13, $289-292$.

Bentтy C. H., Bocek R. M., I97o. Biochemistry of the red and white muscle in the physiology and Biochemistry of muscle as a food, 2, Briskey E. J., Cassens R. G., Marsch B. B. The University of Wisconsin Press, p. I55-207.

Beecher G. R., Cassens R. G., Hoekstra W. G., Briskey E. J., ig65. Red and white fiber content and associated post-mortem properties of seven porcine muscles. $J$. Food Sci., 30, 969-976.

Blanchaer M. C., I964. Respiration of mitochondria of red and white skeletal muscle. Am.J.Physiol., 206, IOI5-1020.

Boccard R., Dumont B. L., I972. Conséquence de l'hypertrophie musculaire héréditaire des bovins sur la musculature. I. Importance relative des différentes régions. Ann. Génét. Sél. anim., 4, I3o.

Briskey E. J., I964. Etiological status and associated studies of pale, soft, exsudative porcine musculature. Adv. Food. Res., 13, 89-178.

Buller A. J., Eccles J. C., Eccles R. M., 1960. Differenciation of fast and slow muscles in the cat hind limb. J. Physiol., 150, 399-416.

Burleigh I. G., Schimke R. T., I968. On the activities of some enzymes concerned with glycolysis and glycogenolysis in extracts of rabbit skeletal muscles. Bioch. Biophys. Res. Comm., 31, 83I-836.

Burleigh I. G., Schimke R. T., I969. The activities of some enzymes concerned with energy metabolism in mammalian muscles of differing pigmentation. Biochem. J., 113, 157-166.

Butrerfield R. M., MaY N. D.S., r966. Muscles of the ox. University of Queensland Press.

Cassens R. G., I970. Summary and discussion of part 2. in The Physiology and Biochemistry of muscle as a food, 2, Briskey E. J., Cassens R. G., MARsch B. B. The university of Wisconsin Press, p. 20g212.

Clarke F. M., Masters C. J., Winzor D. J., I97o. The differential absorption of aldolase isoenzymes in rat brain. Biochem. J, 118, 325-327.

Cooper C. C., Cassens R. G., Kastenschmidt L. L., Briskey E. J., ig7o. Histochemical characterization of muscle differentiation. Develop. Biol., 23, I69-184.

Cooper C. C., Cassens R. G., Kastenschmidt L. L., Briskey E. J., ig7r. Activity of some enzymes in developing muscle of the pig. Pediat. Res., 5, $28 \mathrm{I}-286$.

Cosmos E., r97o. Ontogeny of red and white muscles : the enzymic profil and lipid distribution of immature and mature muscles of normal and dystrophic chickens. in The Physiology and Biochemistry of Muscle as a Food 2, BRiskey E. J., CASsEns R. G., MARsh B. B. The university of Wisconsin Press, p. $193-207$.

Crabtree B., Newsholme E. A., 1972. The activities of phosphorylase, hexokinase, phosphofructokinase, lactate dehydrogenase and glycerol-3-phosphate dehydrogenase in muscles from vertebrates and invertebrates. Biochem. $J$., 126, 49-58.

Dalrymple R. H., Kastenschmidt L. L., Cassens R. G., I973. Glycogen and phosphorylase in developing red and white muscle. Growth $27,19-34$.

Dawson D. M., Romanul F. C. A., 1964. Enzymes in muscle. II. Histochemical and quantitative studies. Arch. Neurology, 11, 369-378.

De Domenech E. M., Domenech C. E., Blanco A., 197o. Distribution of lactate dehydrogenase isozymes in subcellular fractions of rat tissues. Arch. Biochem. Biophys. 141, 147-154. 
Dreyfus J. C., 1967. Sur les différences métaboliques entre les muscles rouges et blancs chez le lapin normal. Rev. Franc. Etudes Clin. Biol, 12, 343-348.

Dubowitz V., 1970. Differentiation of fiber types in skeletal muscle. in The Physiology and Biochemistry of muscle as a food, 2. Briskey E. J., Cassens R. G., Marsh B. B. The University of Wisconsin Press, p. 87-IOI.

Dumont B. L., Boccard R., r972. Conséquences de l'hypertrophie musculaire héréditaire des bovins sur la musculature. II. Importance relative des différents muscles. Ann. Génét. Sél. anim., 4, I30-131.

Durand G., Fauconneau G., Penot E., I966. Croissance des tissus du rat et qualité des protéines alimentaires; influence sur le nombre et la taille des cellules. Ann. Biol, anim. Biochim. Biophys., 6, 389-409.

El-Badawi A. A., Намm R., r972. Aktivität und subcelluläre Verteilung einiger MitochondrienEnzyme im Skeletmuskel. I. Helle und dunkle Muskulatur von Rind und Schwein. A. Lebensmitt., Untersuch. 149, 87-97.

Fox A. C., REED G. E., 1969. Changes in lactate dehydrogenase composition of hearts with right ventricular hypertrophy. Amer. J. Physiol., 216, 1026-1033.

GEYER H., 1968, Isoenzyme der Malatdehydrogenase in tierischen und menschlichen Geweben. $Z$. Analyt. Chemie, 243, 578-586.

Glock G. E., MCLean P., I953. Further studies on the properties and essay of glucose-6-phosphate dehydrogenase and 6-phosphogluconate dehydrogenase of rat liver. Biochem. J., 55, 400-408.

Goldberg A. L., 1967. Protein synthesis in tonic and phasic skeletal muscles. Nature, 216, 1219-I220.

Goldspink G., 1969. Succinic dehydrogenase content of individual muscle fibers at different ages and stages of growth. Life Sci, 8, part II, 79I-808.

Golisch G., Pette D., Pilchmaier H., I97o. Metabolic differentiation of rabbit skeletal muscle as induced by specific innervation. Eur. J. Biochem., 16, I Io-ı I6.

HAмm R., I969. Transaminase of skeletal muscle. II. Transaminase activities in white and red muscles of pigs and cows. J. Food. Sci., 34, 449-452.

Hanset R., ANSEy M., I972. Régions privilégiées d'hypertrophie musculaire, chez le bovin culard. Ann. Méd. Vétér., 116, I7-25.

Hofer S., Hofer H. W., Kuhn E., Pette D., I97I. Changes of hexokinase in the enzyme activity pattern of muscle in human myotonia congenita and in experimental myotonia of the rat. Klin. Wschr., 49, $968-97 I$.

Kraus H., I967. Das Kompensationsphänomen der Glycerin-I-phosphat-oxydase von Pette und Bücher in Skelet- und Herzmuskeln verschiedener Tierklassen (Ratte und Taube). Pfïgers Archiv., 297, 19-26.

Laudahn G., Heyck H., Feustel F., rg68. Enzyme in Serum bei Muskelkrankheiten in Praktisches Enzymologie F. W. Schmidt. Verlag Hans Huber. Bern und Stuttgart, p. 249-3I 7 .

LAWRIE R. A., 1953. The activity of the cytochrome system in muscle and its relation to myoglobin. Biochem. J., 55, 298-305.

MANN W. S., SALAFSKY B., I97o. Enzymic and physiological studies on normal and disused developing fast and slow cat muscles. $J$. Physiol., 208, 33-47.

Mommaerts W. F. H. M., Buller A. J., Seraydarian K., I969. The modification of some biochemical properties of muscle by cross-innervation. Proc. Nat. Acad. Sci. U.S. A., 64, I28-133.

Margreth A., Angelini C., Valfre C., Salviati G., 1970 . Development pattern of LDH isozymes in fast and slow muscles of the rat. Arch. Biochem. Biophys., 141, 374-377.

Mommaerts W. F. H. M., I97o. The role of innervation of the functional differentiation of muscle. in The Physiology and Biochemistry of muscle as a food, 2. BRISKEY E. J., CASSENS R. G., MARSH B. B. The University of Wisconsin Press, p. 53-66.

Moody W. G., Cassens R. G., i968. Histochemical differentiation of red and white muscle fibers. $J$. Anim. Sci., 27, 961-968.

Munro H. N., FLECK A., I966. The determination of nucleic acids in Methods of biochemical analysis, D. GLICK. Interscience Publishers, 14, II3-176.

Opie L. H., Newsholme E. A., 1967. The activities of fructose r.6-diphosphatase, phosphofructokinase and phosphoenolpyruvate carboxykinase in white and red muscle. Biochem. J., 103, $39 \mathrm{I}-399$.

Parsons A. L., Parsons J. L., Blanshard J. M. V., Lawrie R. A., ig69. Electrophoretic differentiation of myofibrillar proteins in the pig. Biochem. $J$., 112, 673-678.

Peter J. B., Jeffress R. N., Lamb D. R., 1968. Exercise : effects on hexokinase activity in red and white skeletal muscle. Science, 160, 200-20I.

Pette D., Luh W., Bucher T., r962. A constant proportion group in the enzyme patterns of the Embden-Meyerhoff chain. Biochem. Biophys. Res. Comm., 7, 419-423.

Pette D., 1966. Mitochondrial enzyme activities in Regulation of metabolic processes in mitochondria, J. M. Tager, S. Papa, E. Quagliariello et E. C. Slater. B. B. A. Library Elsevier, Amsterdam. 7 , $29-49$.

Pette D., r968. Aktivitätsmuster und Ortsmuster von Enzymen des energieliefernden Stoffwechsels in Praktische Enzymologie F. W. Scнмidt. Verlag Hans Huber. Bern und Stuttgart, p. I5-49.

PREwitT M. A., SAlafsky B., I97o. Enzymic and histochemical changes in fast and slow muscles after cross innervation. Amer. J. Physiol., 218, 69-74. 
Ranvier L., I974. De quelques faits relatifs à l'histologie et à la physiologie des muscles striés. Arch Physiol. norm. pathol., 1, 5-15.

Rollins W. C., Julian L. M., Carroll F. D., I969. A note on the body composition of a doublemuscled female and a normal female from a line bred Aberdeen-Angus herd. Anim. Prod., 11, I I I-I I4.

Sarkar S., Sreter F. A., Gergely S., r97I. Light chains of myosins from white, red and cardiac muscle. Proc. Nat. Acad. Sci. U.S. A., 68, 946-950.

Shaw C. R., Prasad R., 1970. Starch gel electrophoresis of enzymes. A compilation of recipes. Biochem. Genetics, 4, 297-320.

Shorr F. A., I969. Protein synthesis by red and white muscle in vitro : effect of insulin and animal age. Am. J. Physiol., 217, 307-309.

Sigel P., Pette D., I969. Intracellular localisation of glycogenolytic and glycolytic enzymes in white and red rabbit skeletal muscle. J. Histochem. Cytochem., 17, 225-237.

Starlinger H., I967. Über die Bindung der Muskelaldolase an Grossdisperse Partikeln in Homogenaten erregter Muskeln. Hoppe-Seyler's Z. Physiol. Chem., 348, 864-87o.

Stein J. M., Padykula H. A., I962. Histochemical classification of individual skeletal muscle fibers of the rat. Amer. J. Anat., 110, I03-123.

Swatland H. J., Cassens R. G., I972. A brief study of muscle enlargement in the rat. J. Anim. Sci., 34, 2 I-24.

Turner L. V., Manchester K. L., I972. Effects of denervation on the glycogen content and on the activities of enzymes of glucose and glycogen metabolism in rat diaphragm muscle. Biochem. J., 128, 789-801.

Wilson A. C., CAHN R. D., Kaplan N. O., I963. Functions of the two forms of lactic dehydrogenase in the breast muscle of birds. Nature, 197, 33I-334.

Wu B. C., Argus M. F., Arcos J. C., 1970. Differential decrease of "Shuttie " enzymes of extramitochondrial $\mathrm{NADH}_{3}$ oxidation of heart muscle during progressive thiamine deficiency. Proc. Soc. Exptl. Biol. Med., 133, 808-812. 